\title{
Influence of structure on the optical limiting properties of nanotubes
}

\author{
N. Izard $^{1,2, *}$, P. Billaud ${ }^{1}$, D. Riehl ${ }^{1}$ and E. Anglaret ${ }^{2}$ \\ 1: Centre Technique d'Arcueil, Délégation Générale pour l'Armement, France \\ 2: Groupe de Dynamique des Phases Condensées, Université Montpellier II, France
}

\begin{abstract}
We investigate the role of carbon nanotube structure on their optical limiting properties. Samples of different and well-characterized structural features are studied by optical limiting and pump-probe experiments. A clear influence of the diameter of the nano-object is demonstrated. Indeed, both nucleation and growth of gas bubbles are expected to be sensitive on diameter. (C) 2021 Optical Society of America
\end{abstract}

OCIS codes: $190.3970,190.4400,190.4870,290.5850$

Nowadays, frequency agile pulsed lasers are widespread. Therefore, development of optical limiting systems, i.e. self-activated protection against potential laser hazard, is an actual challenge. Several non linear optical materials were proposed for optical limiting in the past 15 years, such as reverse saturable absorbers, ${ }^{11}$ multiphoton absorbers ${ }^{2}$ and non linear scattering systems like carbon black suspensions ${ }^{3}$ and single-wall carbon nanotube (SWNT) suspensions ${ }^{[4}$ Indeed, the association of materials with complementarity limiting properties, i.e. non linear scattering from SWNT and multiphoton absorption from organic chromophores, was recently shown to be a promising approach to achieve performant optical limiting systems featuring broad temporal and spectral ranges of efficiency. $\stackrel{[5]}{5}$ The main mechanism responsible for the optical limiting properties of carbon nanotubes suspension is now well known. ${ }^{4}$ Heating of the nanotubes leads to solvent bubbles formation (via heat transfer from nanotubes to solvent) and to sublimation of carbon nanotubes themselves, inducing efficient non linear scattering of the incident laser beam. Optimisation of carbon nanotube suspensions requires a better understanding of relations existing between carbon nanotube structure and their optical limiting properties. Indeed, data currently published are only fragmentary $\stackrel{[6}{7} 8]^{\text {Either }}$ materials studied are not well characterized, or studies concern only a few samples.

In this letter, we report on the optical limiting behavior of model carbon nanotubes of various structures. Samples were purchased from MER Inc, Nanoledge and Nanolab Inc. SWNT were produced by the electric arc process while MWNT were produced by a CVD 
process. All samples were extensively characterized using scanning and transmission electron microscopy, X-Ray diffraction, Raman and optical spectroscopy 9 The mean diameter of SWNT is $1.35 \pm 0.15 \mathrm{~nm}$. Nanotubes assemble into crystalline hexagonal bundles, with a diameter of about 10 to $15 \mathrm{~nm}$. We also worked on MWNT samples of different length and diameter distributions, as summarized in table 1.

Aqueous suspensions of nanotubes were realised from these raw samples in water, using 1 wt\% of Sodium Dodecyl Sulfate (SDS), and will be designated thereafter SWNT-Bundle, MWNT-1... A suspension of individualised SWNT was also prepared from the suspensions of SWNT bundles, following the procedure described by O. Connel et al. in reference10, and will be designated SWNT-Individual. Evidence of SWNT exfoliation was obtained by direct HRTEM observation, fluorescence and Raman scattering!11 At last, we prepared a suspension of SWNT shortened by an oxidative treatment, designated SWNT-Short. We checked by electron microscopy that these tubes were significantly shorter (below $100 \mathrm{~nm}$ ) than the unshortened tubes $(>1 \mu \mathrm{m})$ and that the diameter of the bundle was not affected (not shown). Linear optical transmissions of the suspensions were adjusted to $70 \%$ at $532 \mathrm{~nm}$ in $2 \mathrm{~mm}$ thick cells (concentration was around $10 \mathrm{mg} / \mathrm{l}$ ). Non linear optical transmittance measurements were performed using a Q-switched, but non injected, frequency doubled Nd:YAG, with a pulse duration of $15 \mathrm{~ns}$ in a F/50 focusing geometry. Pump-probe experiments were performed using a frequency doubled Nd:YAG pump laser emitting $4 \mathrm{~ns}$ pulses at $532 \mathrm{~nm}$ and a $633 \mathrm{~nm}$ continuous probe.

We first investigated the influence of nanotube length. Optical limiting measurements of the suspensions MWNT-1/5 are reported on figure $1 \mathrm{a}$. Optical limiting performances are comparable for all samples, despite the strong variation in nanotube length (there is a factor 5 between the longest and the shortest tube). This result clearly states that nanotube length is not a structural parameter influencing on the optical limiting properties. No diameter influence was observed neither, but here the diameter variation is small (factor 2 at most). Optical limiting properties of suspensions SWNT-Bundle and SWNT-Short, which contain nanotubes of same diameter are reported on figure $1 \mathrm{~b}$. These data confirm the non influence of the length. Note that Riggs et al.12] reported slightly weaker optical limiting performances for shortened nanotubes, but their data cannot assign it to a length effect : such a behavior could also be due to a slight unbundling of the nanotubes consecutive to the shortening process, and the lack of pre- and post-process characterization of nanotube diameter and length do not allow to choose between the two hypotheses.

We also studied the influence of nanotube diameter. Figure 2 presents transmittance measurements for suspensions of individual SWNT, bundled SWNT and MWNT. The diameter of MWNT $(20-50 \mathrm{~nm})$ is larger than the diameter of bundled SWNT $(10-15$ $\mathrm{nm})$, itself larger than the diameter of individualised SWNT $(1.4 \mathrm{~nm})$. The optical limita- 
tion thresholds obtained with MWNT, bundled SWNT and individual SWNT suspensions are around $100 \mathrm{~mJ} \cdot \mathrm{cm}^{-2}, 200 \mathrm{~mJ} \cdot \mathrm{cm}^{-2}$ and $400 \mathrm{~mJ} \cdot \mathrm{cm}^{-2}$, respectively. The dependency of the optical limiting properties on the nano-object diameter is thus clearly demonstrated. The better efficiency, and the lower optical limiting threshold, are achieved for the largest diameter of the nano-object.

To get a better insight on the effect of the diameter, we carried out pump-probe experiments on SWNT suspensions, in bundles (Fig. 3 a ) and individualised (Fig. 3 b ). At low fluence, $52 \mathrm{~mJ} \cdot \mathrm{cm}^{-2}$ for SWNT-Bundle suspension, the probe is not perturbated by the pump.

The fluence of $150 \mathrm{~mJ} \cdot \mathrm{cm}^{-2}$ corresponds roughly to the limitation threshold : the probe is perturbed (i.e. bubbles are created) just at the end of the pump pulse. This threshold value is the limiting threshold, which is comparable to the value determined from figure 2 although the pulse is shorter. At last, for higher pump energy, $540 \mathrm{~mJ} \cdot \mathrm{cm}^{-2}$, the transmission of the probe falls at the beginning of the pump pulse : the transferred energy is sufficient to sublimate nanotubes and to create bubbles effective for optical limiting. The behavior is dramatically different for SWNT-Individual suspensions. Indeed, at $150 \mathrm{~mJ} \cdot \mathrm{cm}^{-2}$, probe transmission falls slightly more than 10 ns after the passing of the pump. This means that some bubbles are nucleated inside the suspension by the pump, but they are too small to be effective for limiting. Fluence must increase up to $540 \mathrm{~mJ} \cdot \mathrm{cm}^{-2}$ to reach the limitation threshold. At $4500 \mathrm{~mJ} \cdot \mathrm{cm}^{-2}$ an efficient optical limiting of the pump beam is observed. This value is more than 8 times larger for individual tubes than for bundles.

We attribute this result to the effect of diameter on nucleation and growth of bubbles. Indeed, it is reasonable to assume than the size of the scattering center created by the nano-object is a function of its diameter. Consequently, individualised nanotubes are expected to nucleate smaller bubbles than bundled nanotubes. This observation leads to two consequencies. From the Laplace law (here below given for a sphere),

$$
P_{\text {ext }}=P+\frac{2 \gamma}{R}
$$

where $P$ is the pressure of a gas bubble, $P_{\text {ext }}$ the pressure of the surrounding fluid, $\gamma$ the tensile surface liquid / gas and $R$ the radius of a bubble, the surpression needed to nucleate a bubble in a liquid increases when its diameter decreases. So, more energy is involved in the nucleation of bubbles in individual tube suspensions than for bundled tube suspensions, i.e. bundled tube will create bubbles at lower incident fluence than individuals. Furthermore, once nucleated, the diameter of the bubble is much smaller than the laser wavelength, so bubbles will scarcely scatter the incident laser beam. They have to grow until they reach a "critical" (efficient for scattering) size allowing efficient light scattering. This growth time strongly depends on liquid / gas tensile surface and other thermodynamical parameters, 13 
but also on the initial bubble size. Thus, a small bubble will take a longer time to reach the "critical" size than a larger bubble. Therefore, suspensions of individualised nanotubes will present a poorer optical limiting efficiency than suspensions of bundled nanotubes.

Finally, it cannot be ruled out that nanotubes in bundles heat faster than individualised tubes. This will occur if the heat capacity for bundles is smaller than that for individualised tubes or if absorption cross section per unit of mass is larger for bundles than for individual. Data on nanotube thermal properties are scarce and did not make distinction between tubes in bundles or individualised. Meanwhile, plasmons are mainly responsible for absorption in carbon nanotubes, and their properties are expected to be sensitive on environment (including bundling). To go further in the interpretation, plasmon coupling between carbon nanotubes inside the bundles may induce a significant absorption enhancement.

In summary, we reported a clear influence of the nanotube diameter on their optical limiting properties. A two-fold interpretation is proposed. First, a larger nanotube size involves a larger nucleation center and a faster growth and then improves optical limiting efficiency. Second, the absorption cross section might be larger for bundled nanotubes, due to changes in plasmon properties. In conclusion, increasing nanotube diameter will help improving the optical limiting properties of suspensions. However, the increase in size must be limited in order to preserve the stability of suspensions, and to prevent from light scattering at low fluences. Anyway, this approach is interesting and promising, and is another step towards the realisation of laser protection for the human eye.

We acknowledge E. Doris and C. Ménard for fruitful discussions and their useful advice for the preparation of the samples.

*: Email: izard@gdpc.univ-montp2.fr

\section{References}

1. W.F. Sun, C.C. Byeon, C.M. Lawson, G.M. Gray and D.Y. Wang, Applied Physics Letter 77 (2000) 1759.

2. G.S. He, G.C. Xu, P.N. Prasad, B.A. Reinhardt, J.C. Bhatt, R. McKellar and A.G. Dillard, Optics Letters 20 (1995) 435.

3. K. Mansour, M.J. Soileau and E.W. Van Stryland, Journal of the Optical Society of America B 9 (1992) 1100.

4. L. Vivien, P. Lançon, D. Riehl, F. Hache and E. Anglaret, Carbon 40 (2002) 1789.

5. N. Izard, C. Ménard, D. Riehl, E. Doris, C. Mioskowski and E. Anglaret, Chem. Phys. Lett. 391 (2004) 124.

6. C. Li, C. Liu, F. Li and Q. Gong, Chem. Phys. Lett 380 (2003) 201.

7. Z. Hongbing, C. Wenzhe, W. Minquan, Zhengchan and Z. Chunlin, Chem. Phys. Lett. $382(2003) 313$. 
8. J. Xu, M. Xiao, R. Czerw and D.L. Carroll, Chem. Phys. Lett. 389 (2004) 247.

9. N. Izard, D. Riehl and E. Anglaret, AIP Conference Proceedings, vol. 685, Molecular Nanostructures, 2003, p. 235.

10. M.J. O'Connel, S.M. Bachilo, C.B. Huffman, V.C. Moore, M.S. Strano, E.H. Haroz, K.L. Rialon, P.J. Boul, W.H. Noon, C. Kittrell, J. Ma, R.H. Hauge, R.B. Weisman and R.E. Smalley, Science 297 (2002) 593.

11. N. Izard, D. Riehl and E. Anglaret, Phys. Rev. B, in press.

12. J.E. Riggs, D.B. Walker, D.L. Carroll and Y.-P. Sun, J. Phys. Chem. B 104 (2000) 7071.

13. L. Vivien, D. Riehl, J. Delouis, J.A. Delaire, F. Hache and E. Anglaret, Journal of the Optical Society of America B 19 (2002) 208. 
Table 1. Length and tube diameter distributions for MWNT samples

\begin{tabular}{ccc}
\hline Sample & Tube diameter $(\mathrm{nm})$ & Length $(\mu \mathrm{m})$ \\
\hline MWNT-1 & $20-50$ & $5-20$ \\
MWNT-2 & $20-50$ & $1-5$ \\
MWNT-3 & $10-20$ & $5-20$ \\
MWNT-4 & $10-20$ & $1-5$ \\
MWNT-5 & $10-20$ & $<1$ \\
\hline
\end{tabular}


Fig. 1: Normalized transmittance measurements with $15 \mathrm{~ns}$ pulses at $532 \mathrm{~nm}$ for (a) MWNT-1 to MWNT-5 suspensions (b) SWNT-Bundle and SWNT-Short suspensions.

Fig. 2: Normalized transmittance measurements with $15 \mathrm{~ns}$ pulses at $532 \mathrm{~nm}$ for suspensions SWNT-Individual, SWNT-Bundle and MWNT-1.

Fig. 3: Pump-probe experiments for suspensions of (a) SWNT-Bundle and (b) SWNTIndividual. In each case, pump profile (532 nm, $4 \mathrm{~ns}$ ) is represented by a plain line, while the probe profile $(633 \mathrm{~nm})$ is represented by a dashed line for several pump energies. 

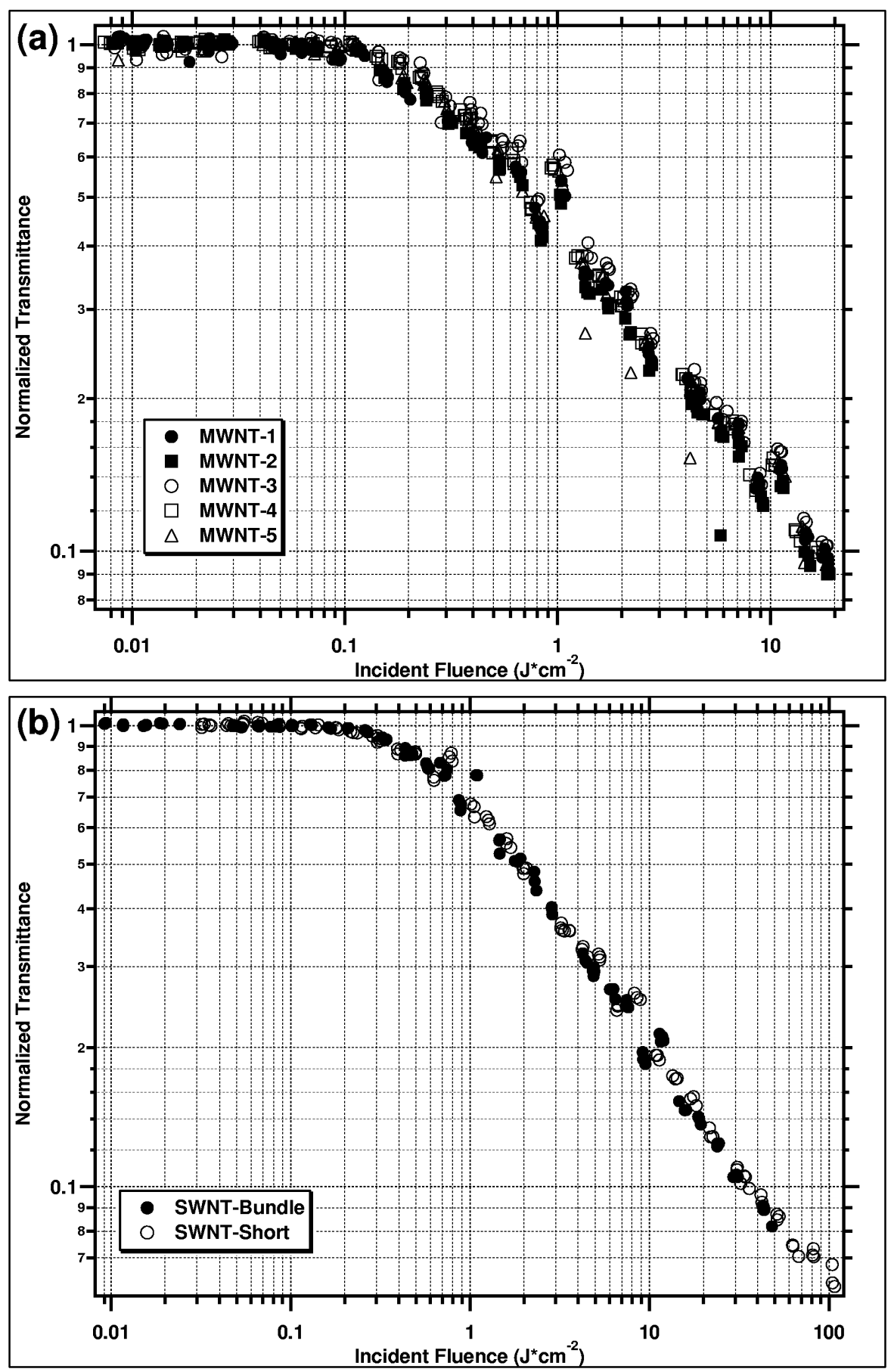

Fig. 1. 


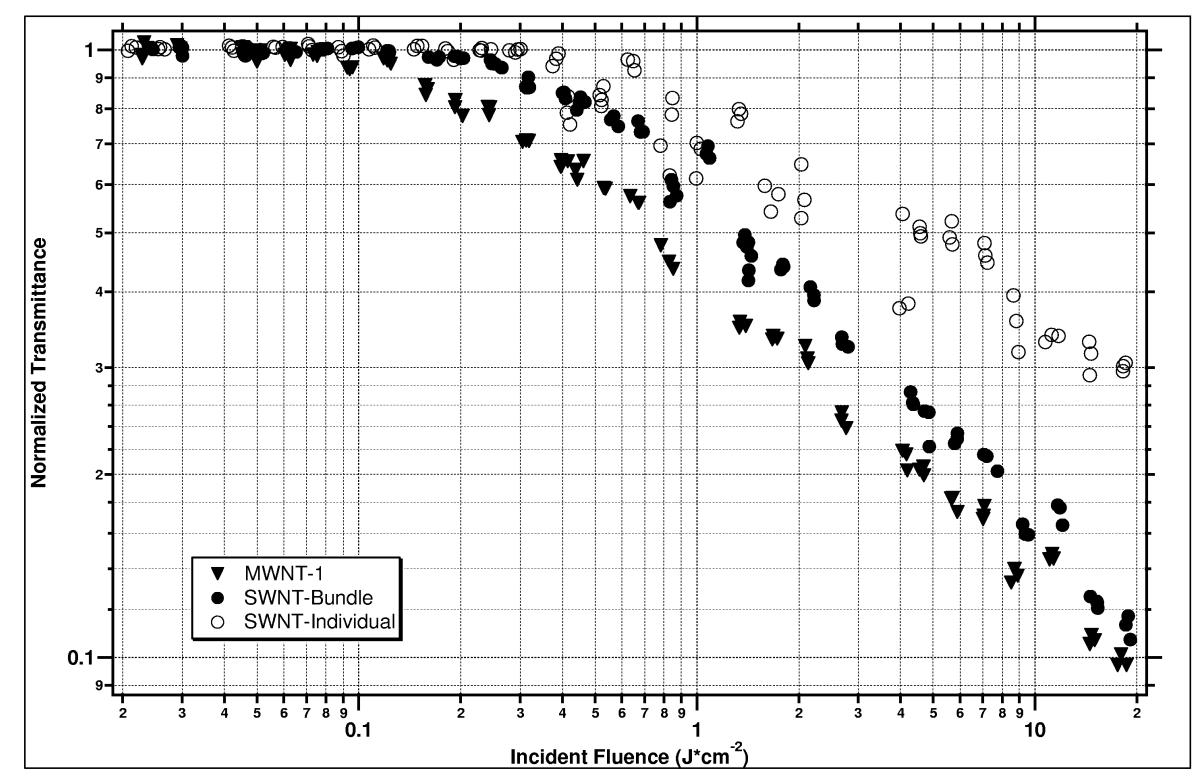

Fig. 2. 

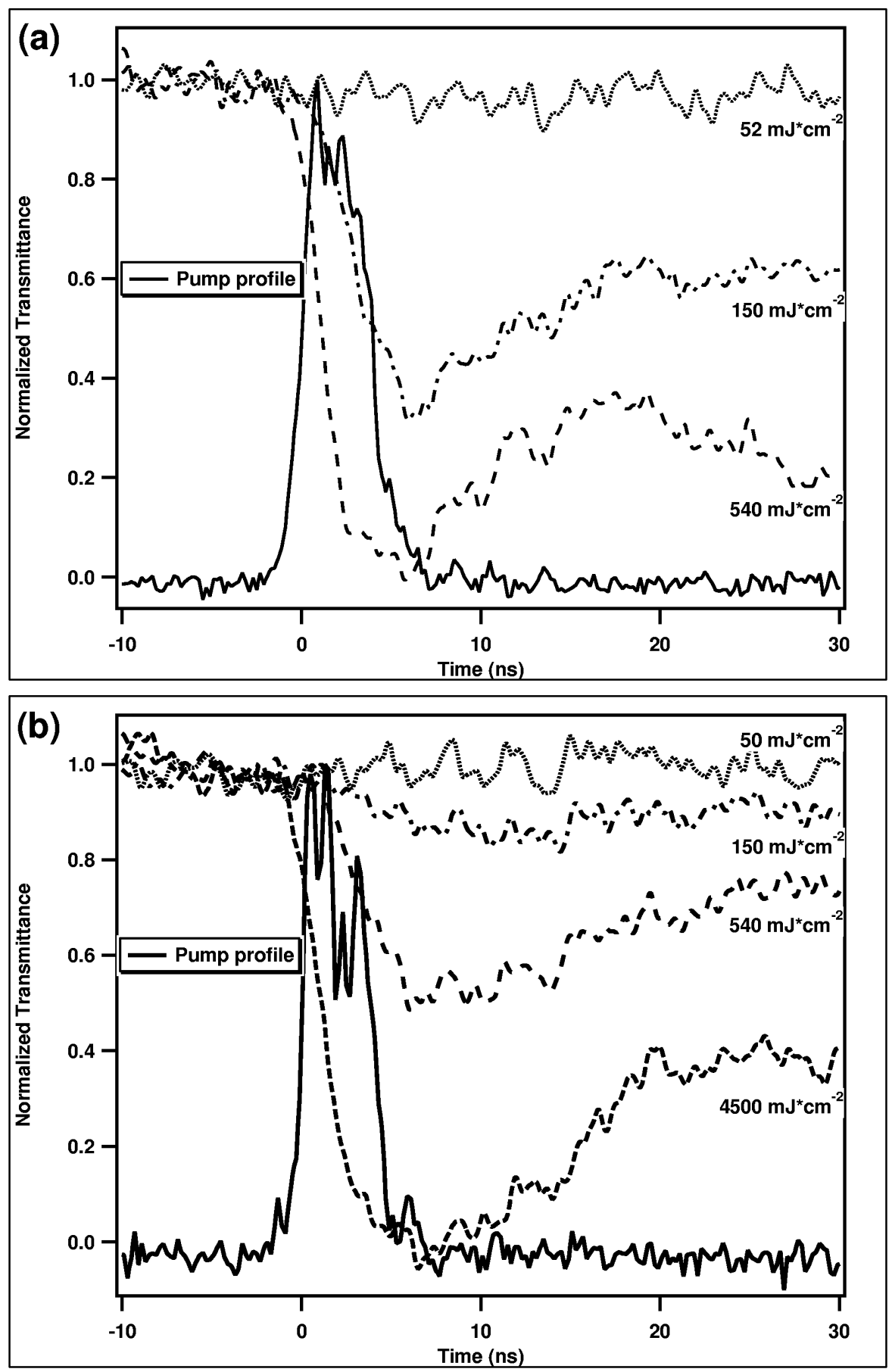

Fig. 3. 